\title{
Evidence for a palmoplantar-specific role for microtubules
}

Hélène Louis dit Picard ${ }^{1}$, Diana C. Blaydon ${ }^{1}$, David P. Kelsell ${ }^{1}$

${ }^{1}$ Centre for Cutaneous Research, Blizard Institute, Barts and the London School of Medicine and Dentistry, Queen Mary University of London, UK

\section{Introduction}

- The palmoplantar epidermis is uniquely adapted to withstand physical stress

- Investigating cytoskeleton and microtubules in palm using palmoplantar keratoderma models and two proteins AQP5 and iRhom2 that appear to have key roles in regulating these key cellular processes

\section{Results}

Microtubule dynamics differ between interfollicular and palmoplantar skin
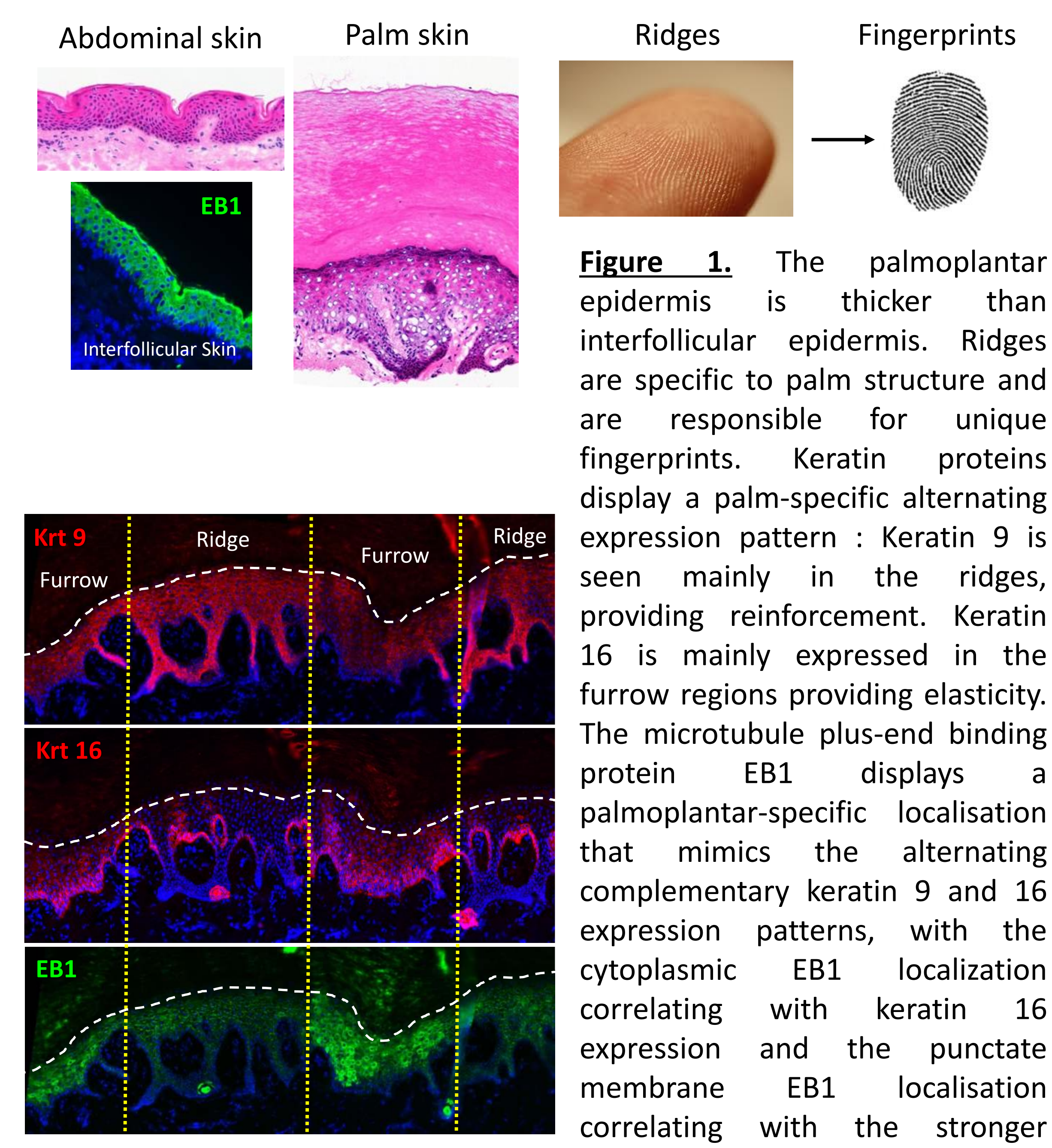
epidermis is the palmoplantar interfollicular epidermis. Ridges are specific to palm structure and are responsible for unique fingerprints. Keratin proteins display a palm-specific alternatin seen mainly in the ridges, providing reinforcement. Keratin 16 is mainly expressed in the furrow regions providing elasticity. The microtubule plus-end binding protein EB1 displays palmoplantar-specific localisation that mimics the alternating expression patterns, with the cytoplasmic EB1 localization correlating with keratin 16 membrane EB1 localisation correlating with the stronger keratin 9 expression.
AQP5 mutations leads to microtubule dysregulation
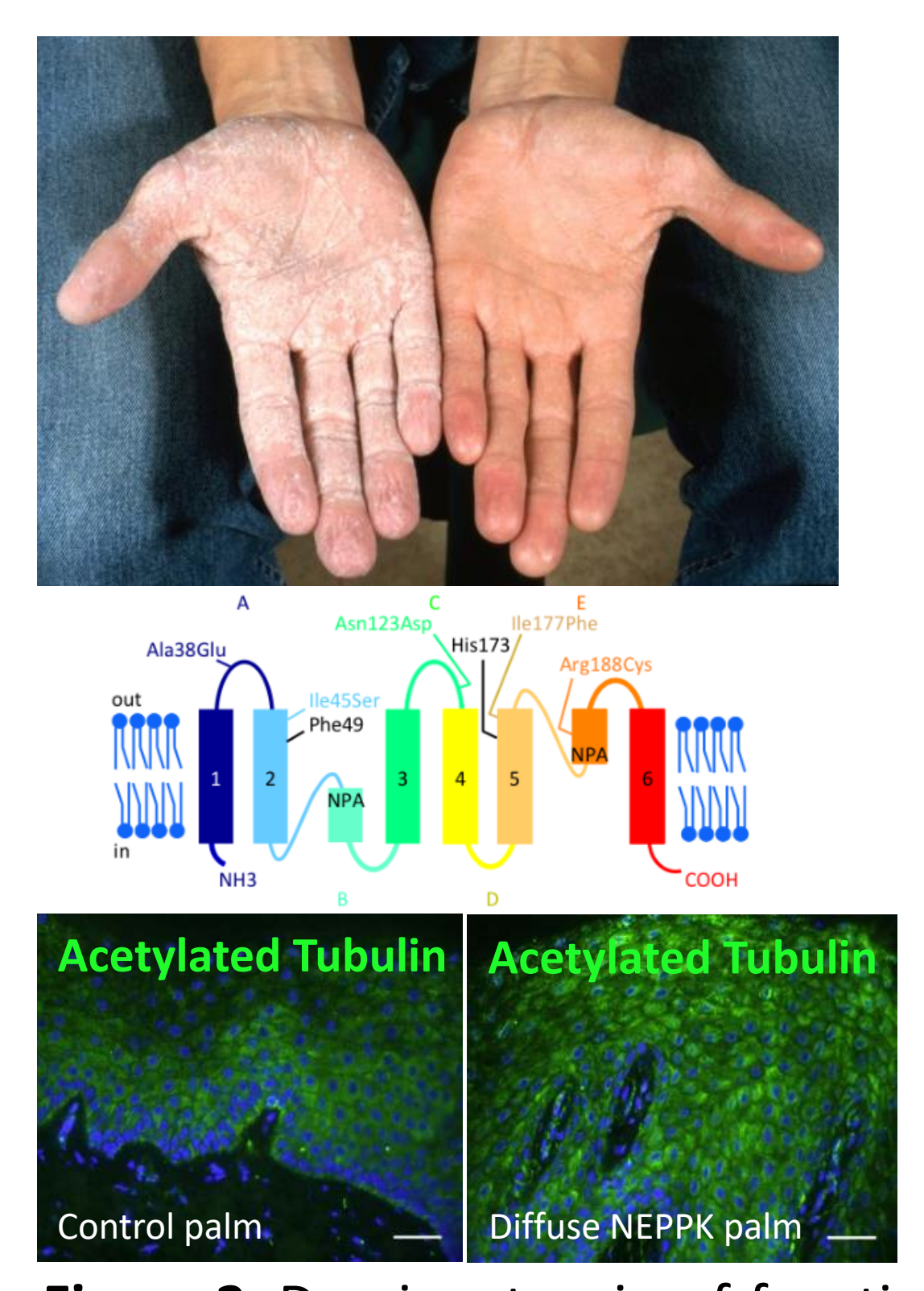

Figure 2. Dominant, gain-of-function mutations in AQP5 underlie diffuse NonEpidermolytic Palmoplantar Keratoderma (dNEPPK). Patients palm skin revealed rapid and transient formation of edematous whitish plaques on the palms on exposure to water. AQP5 is widely expressed in the sweat gland and patients are reported to show excessive sweating. AQP5 reported to be able to enhance microtubule organization and stability in airway epithelial cells. Palm skin harbouring AQP5 mutation exhibits increased levels of acetylated tubulin, a marker for microtubule stabilization, compared to control palm skin. Desmoplakin is reported to interacts with EB1 and the interaction between desmoplakin and EB1 is central to the ability of desmoplakin to regulate microtubule dynamics. dNEPPK palm skin displays a predominantly membrane localisation for EBI and almost complete loss of the cytoplasmic EB1 localisation, further evidence that AQP5 may play a role in regulating microtubule dynamics in palm skin.
iRhom 2 regulates the cytoskeleton and the microtubule network

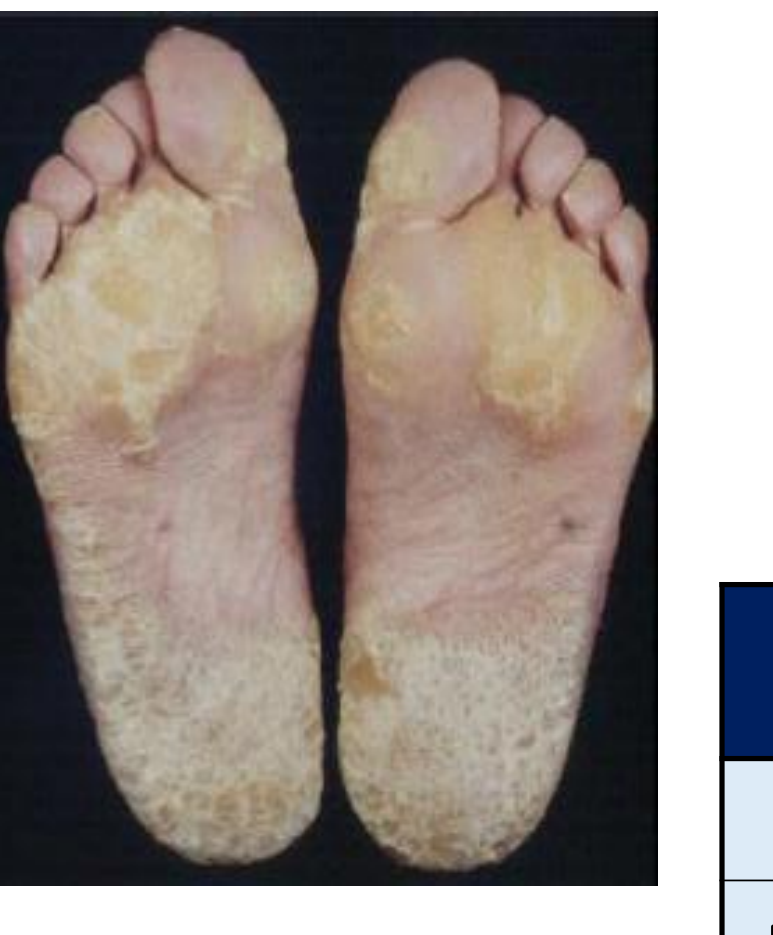

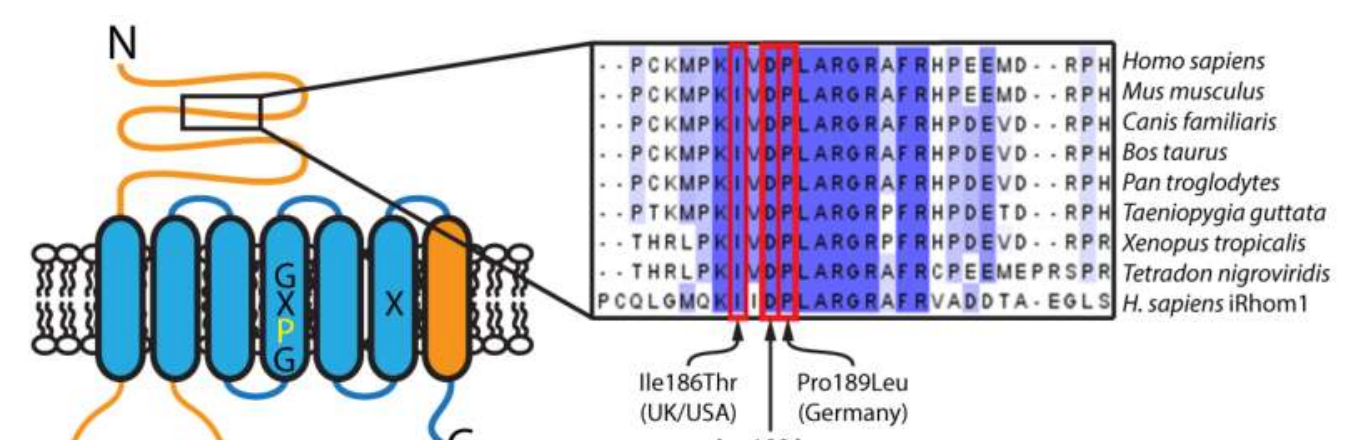

\begin{tabular}{|c|c|}
\hline Protein Class & iRhom2-Interacting Proteins \\
\hline Filament Protein & KRT14, KRT16, KRT17 \\
\hline Microtubule Stability & MACF1, LZTS2, CEP70, TACC2, PDE4DIP \\
\hline Cytoskeletal Dynamics & BPAG1, HSPB3 \\
\hline
\end{tabular}
\begin{tabular}{l|l} 
Cytoskeletal Dynamics & BPAG1, HSPB3 \\
\hline
\end{tabular} Figure 3. IRHOM2 mutations are responsible for

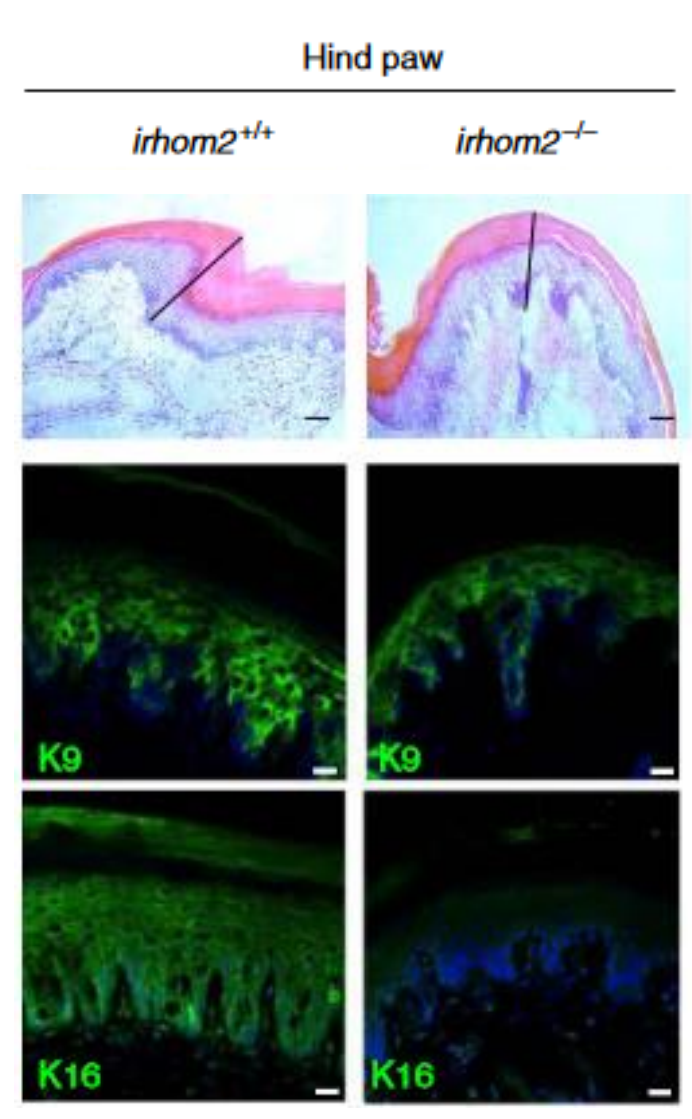
evealed focal thickening of palms and soles. All the dominant, gain-of-function mutations in iRHOM2 are identified in the cytoplasmic $\mathrm{N}$-terminus domain. Using a yeast-2-hybrid (Y2H) screen with iRhom2 as bait, we identified the major cytoskeletal stress keratin, K16, as an interacting binding partner of iRhom2. We showed a higher expression of K16 in TOC skin compared to control and by studying the irhom $2^{--}$mice model, we identified a reduced K16 expression compared to control revealing that $\mathrm{K} 16$ expression is regulated by iRhom

The $\mathrm{Y} 2 \mathrm{H}$ study allowed us to identify different proteins related to the microtubule stability and the cytoskeletal dynamics possibly linked to iRhom2.

iRhom2 cytoplasmic N-terminus interacts with two giant cytoskeletal crosslinkers : BPAG1 and MACF1

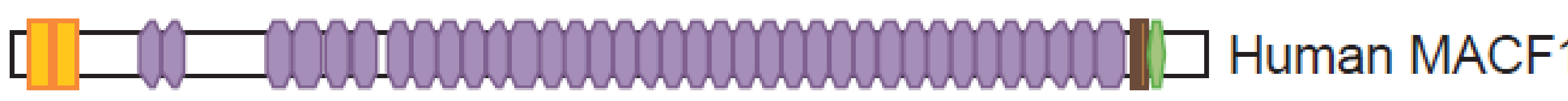

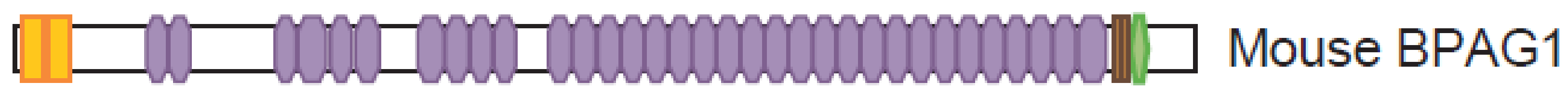

- Regulation of actin cytoskeleton

- Stability of the cytoskeletal structure

\begin{tabular}{|l|l|}
\hline $\begin{array}{l}\text { Calponin homology } \\
\text { Spectrin repeat }\end{array}$ & EF hand \\
\hline
\end{tabular}

- Focal adhesion
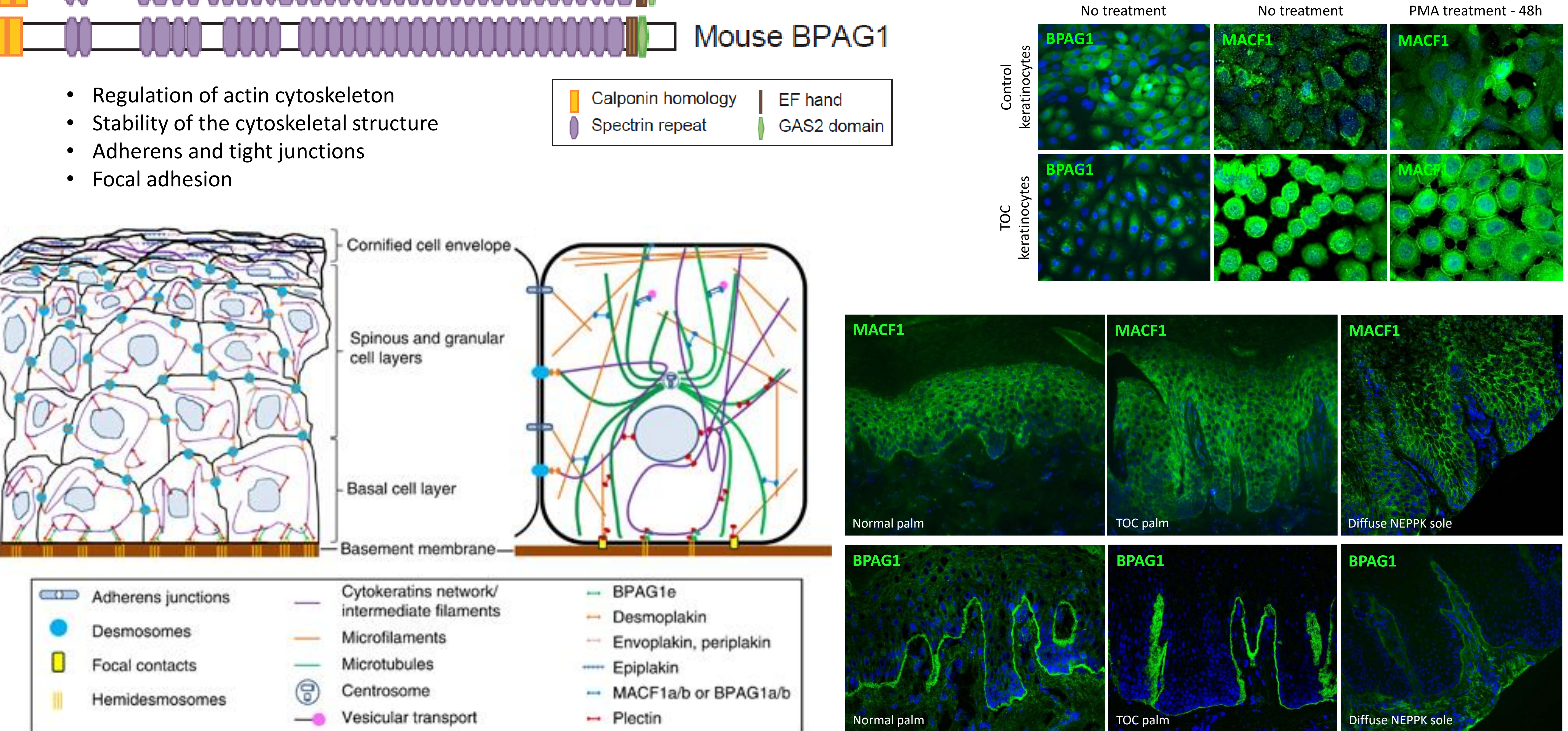

Figure 4. We report BPAG1 (Bullous Pemphigoid Antigen 1 or Dystonin) and MACF1 (MicrotubuleActin Crosslinking Factor 1 or ACF7) as iRhom2 interacting partners, via their spectrin repeat domain. These are both spectraplakins; giant cytoskeletal linker proteins that can interact with all three cytoskeletal components: actin, microtubules and intermediate filaments. The spectrin repeat domain is involved in cytoskeletal architecture and in forming large signal transduction complexes. We show different expression localisation of BPAG1 expression in TOC patient-derived keratinocytes compared to controls. We showed a higher expression of MACF1 in TOC patient-derived keratinocytes compared to controls. When we stimulated these cells with PMA inducing differentiation processes, we identified a stronger MACF1 expression at the cell surface in TOC patientderived keratinocytes.

We show increased levels of BPAG1 in TOC palm at the epidermal-dermal junction and in addition MACF1 displays increased membrane localisation in dNEPPK palmoplantar epidermis. MACF1 and BPAG1 are EB1 interacting protein and EB1 also displayed increased membrane localisation in dNEPPK palmoplantar.

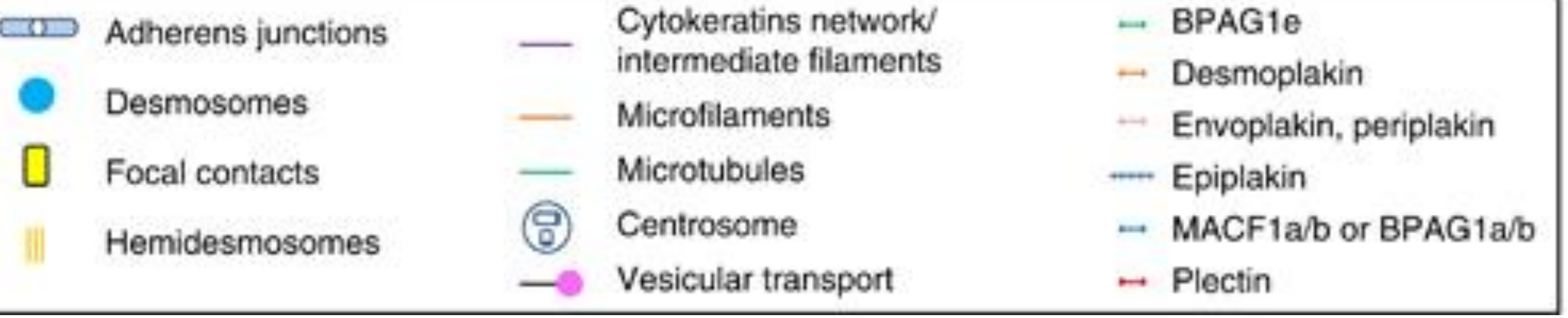

Conclusion - Perspectives

- We report BPAG1 (Bullous Pemphigoid Antigen 1 or Dystonin) and MACF1 (Microtubule-Actin Crosslinking Factor 1 or ACF7) as iRhom2 interacting partners, via their spectrin repeat domain. These are both spectraplakins; giant cytoskeletal linker proteins that can interact with all three cytoskeletal components

- Immunostaining revealed a palmoplantar-specific localisation pattern for the microtubule plus-end binding protein, EB1, that correlated with the palmoplantar ridges and also the alternating expression patterns for keratins 9 and 16, indicating that microtubule dynamics differ between interfollicular and palmoplantar skin - Whilst the physical resilience of the palmoplantar epidermis has been linked predominantly to an abundance of keratin intermediate filaments, we have now identified a potential role for microtubules in this function 